\title{
Simulations and Experiments on the Vibrational Characteristics of Cylindrical Shell Resonator Actuated by Piezoelectric Electrodes with Different Thicknesses
}

\author{
Yiming Luo, Tianliang Qu, Bin Zhang, Yao Pan, and Pengbo Xiao \\ College of Optoelectronic Science and Engineering, National University of Defense Technology, Changsha, China
}

Correspondence should be addressed to Tianliang Qu; qutianliang@nudt.edu.cn

Received 24 March 2017; Accepted 15 May 2017; Published 12 June 2017

Academic Editor: Athanasios Chasalevris

Copyright (C) 2017 Yiming Luo et al. This is an open access article distributed under the Creative Commons Attribution License, which permits unrestricted use, distribution, and reproduction in any medium, provided the original work is properly cited.

\begin{abstract}
The resonator is the key element of the Coriolis Vibratory Gyroscope (CVG). The vibrational characteristics of the resonator, including the resonant frequency, vibrational amplitude, and $Q$ factor, have a great influence on CVG's performance. Among them, the vibrational amplitude mainly affects the scale factor and the signal-to-noise ratio, and the $Q$ factor directly determines the precision and drift characteristics of the gyroscope. In this paper, a finite element model of a cylindrical shell resonator actuated by piezoelectric electrodes with different thicknesses is built to investigate the vibrational characteristics. The simulation results indicate that the resonant frequency barely changes with the electrode thickness, whereas the vibrational amplitude is inversely proportional to the electrode thickness under the same driving voltage. Experiments were performed with four resonators and piezoelectric electrodes of four sizes, and results were consistent with simulations. The resonant frequencies of four resonators changed within $0.36 \%$ after attaching the piezoelectric electrodes. Meanwhile, with the same driving voltage, it was shown that the vibrational amplitude decreased with the increase of electrode thickness. Moreover, thinner electrodes resulted in better $Q$ factor and therefore better performance. This study may provide useful reference on electrode design of the CVGs.
\end{abstract}

\section{Introduction}

The cylindrical resonator gyroscope $(\mathrm{CRG})$ is a kind of Coriolis Vibratory Gyroscope (CVG) which measures angular velocity through the precession of elastic waves. The CRG has many advantages, such as high reliability, high stability, long life, low power consumption, and small size, and is widely used in the medium- and low-precision navigation fields and platform stabilization systems [1-6]. The representative products include the Watson Inc. Pro Gyro ${ }^{\circledR}$ series [7] and the InnaLabs Inc. GI-CVG series [8]. The cylindrical shell resonator is the core element of the CRG, the vibrational characteristics of which determine its overall performance.

Normally, the resonator can maintain the maximum amplitude at the resonant frequency. A higher amplitude is advantageous in scale factor and detecting signals, which brings a better signal-to-noise ratio (SNR) [9-12]. Q factor directly determines the precision and drift characteristics of gyroscopes [13]. Pan et al. reported a fused silica cylindrical resonator with the $Q$ factor approaching 1000000 [14]. However, when actuated by piezoelectric electrodes (also called piezoelectrodes) [15], the $Q$ factor dropped dramatically. Therefore, it is of great importance to study the vibrational characteristics of resonators with attached piezoelectrodes.

To our knowledge, there was no relevant research on how the thickness of piezoelectrodes influences the vibrational characteristics such as resonant frequency, vibrational amplitude, and $Q$ factor.

In this paper, we first presented the mathematical model of the resonator actuated by piezoelectrodes. Then, we built a finite element model through ANSYS to investigate its vibration shape, resonant frequency, and vibrational amplitude of the resonator with attached piezoelectrodes. Finally, we performed experiments on four resonators made of brass and piezoelectrodes made of PZT-5H. Experimental results were consistent with simulations. We demonstrated that thinner electrodes introduce less degradation of $Q$ factors and meanwhile provide a larger amplitude with the same 
TABLE 1: Parameters of materials and piezoelectric constants of PZT-5H.

\begin{tabular}{lcccc}
\hline Component & Material & Poisson's ratio & Young's modulus $(\mathrm{GPa})$ & \multicolumn{1}{c}{ Density $\left(\mathrm{Kg} / \mathrm{m}^{3}\right)$} \\
\hline Resonator & Brass & 0.33 & 110 & 8430 \\
Piezoelectrodes & PZT-5H & 0.31 & 70 & 7800 \\
\hline Physical quantities & $c_{13}\left(\mathrm{~N} / \mathrm{m}^{2}\right)$ & $c_{33}\left(\mathrm{~N} / \mathrm{m}^{2}\right)$ & $c_{11}\left(\mathrm{~N} / \mathrm{m}^{2}\right)$ & $e_{33}\left(\mathrm{C} / \mathrm{m}^{2}\right)$ \\
Numeric & $9.1 \times 10^{10}$ & $13.2 \times 10^{10}$ & $14.9 \times 10^{10}$ & 17.5 \\
\hline
\end{tabular}

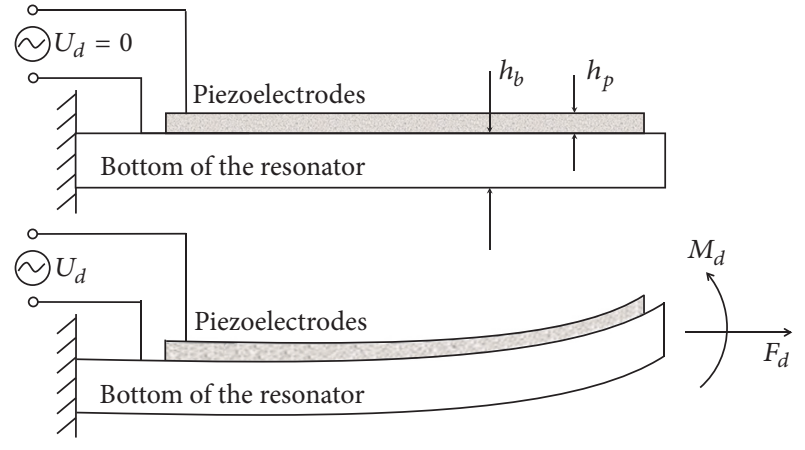

FIGURE 1: Schematic diagram of piezoelectrodes.

driving voltage, which is preferred for the SNR of the gyroscope.

\section{Mathematical Models}

This paper describes a kind of resonators, including a resonant shell, a vibration-conducting shell, a bottom plate, and a stem. Eight piezoelectrodes are attached on the bottom of the resonator symmetrically to actuate the resonator and detect its vibrational signal, as shown in Figure 2. The resonator is actuated by piezoelectrodes to its lowest bending mode, also called the wineglass mode, with 2 degenerate modes that are spatially orthogonal with each other. Usually, The CVG works in the force-to-rebalance mode, in which two driving piezoelectrodes actuate the resonator into the wineglass mode and maintain a stable amplitude, and the Coriolis force stimulates the other degenerate mode. The controlling piezoelectrodes produce a signal to null the secondary mode, the amplitude of which is linearly proportional to the angular rate [16-19].

The piezoelectrodes were attached on the bottom of the resonator. A simplified model is shown in Figure 1. When the voltage is exerted on the piezoelectrodes, the driving force $F_{d}$ and the driving torque $M_{d}$ will act on the resonator. The intensity of $F_{d}$ and $M_{d}$ depend on the driving voltage, the piezoelectric properties, geometric shape, and size of the piezoelectrodes. The driving force $F_{d}$ and the driving torque $M_{d}$ can be expressed as [20-23]

$$
\begin{aligned}
F_{d}= & \left(\frac{c_{13} e_{33}}{c_{33}}-e_{31}\right) U_{d} b_{p}+\left(c_{11}-\frac{c_{13}^{2}}{c_{33}}\right) S_{1} b_{p} h_{p}, \\
M_{d}= & \left(\frac{c_{13} e_{33}}{c_{33}}-e_{31}\right) U_{d} b_{p} \frac{h_{b}+h_{p}}{2} \\
& +\left(c_{11}-\frac{c_{13}^{2}}{c_{33}}\right) S_{1} b_{p} h_{p} \frac{h_{b}+h_{p}}{2}
\end{aligned}
$$

where $U_{d}$ is the driving voltage, $S_{1}$ is the weight of strain tensor $S$ of the piezoelectrodes at the radial direction, $b_{p}$ is the width of the piezoelectrodes, $h_{p}$ is the thickness of the piezoelectrodes, $h_{b}$ is the thickness of the bottom of the resonator, and $c_{11}, c_{13}, c_{33}, e_{31}$, and $e_{33}$ are the weight of the coefficient in the parameter matrix of PZT-5H.

For the cylindrical shell resonator, $Q$ factor refers to the ratio of the energy loss in a vibrational cycle to the total energy stored in the resonator, which can be expressed as [24]

$$
Q=\frac{2 \pi \mathrm{E}}{\Delta E}=\frac{2 \pi E}{\sum_{i=1}^{n} \Delta E_{i}},
$$

where $E$ refers to the total energy stored in the resonators and $\Delta E_{i}$ refers to the energy loss in one cycle caused by No. $i$ th factor.

As simulations on the $Q$ factor are complicated, this paper investigates the $Q$ factor degradation with the thickness of piezoelectrodes in experiment. The $Q$ factor was measured and calculated through MATLAB by the ring-down method, which can be expressed as

$$
Q=\pi f \tau,
$$

where $f$ is the resonant frequency and $\tau$ is the decay time, meaning the time during which the vibrational amplitude decreased to its $1 / e$.

\section{Results and Discussion}

3.1. Simulation. When the resonator is at resonance, the steady-state vibrational amplitude depends on the driving force and the torque induced by the piezoelectrodes, which are related to the geometric dimension of the piezoelectrodes. However, the numerical equations cannot describe the correlation between them clearly. Therefore, a finite element model was built with ANSYS. The parameters of materials and the piezoelectric constants of PZT-5H were shown in Table 1. In the finite element model, the thicknesses of the piezoelectrodes were changed from $100 \mu \mathrm{m}$ to $800 \mu \mathrm{m}$ with an increment of $100 \mu \mathrm{m}$. The length and width of the piezoelectrodes were $8 \mathrm{~mm}$ and $1 \mathrm{~mm}$, respectively. The distances between the geometric centers of the piezoelectrodes and the center of the resonator were $9 \mathrm{~mm}$. Other geometric dimensions of the resonator are shown in Table 2 and Figure 2. The driving voltages were set at $10 \mathrm{~V}$ throughout the simulation. In addition, according to Zhu et al. [25], the epoxy resin adhesives have minor influence on the vibrational characteristics; therefore we did not include epoxy resin adhesive in our model. This simulation demonstrated how the resonant frequency and the amplitude change with the thickness of piezoelectrodes, so as to find an optimal thickness 


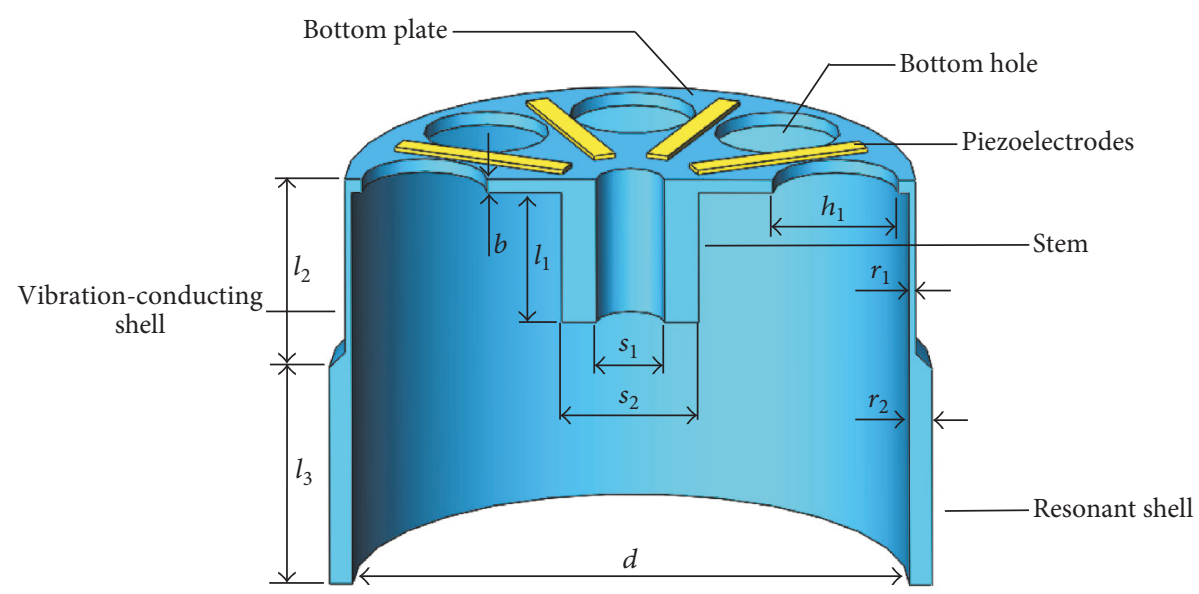

FIGURE 2: The simulation structure of the resonator.

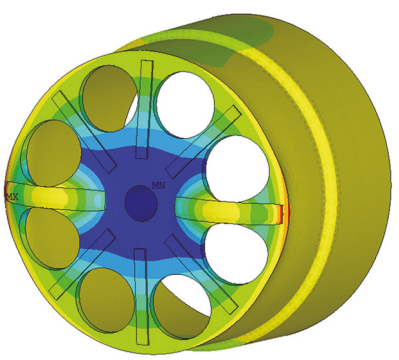

$200 \mu \mathrm{m}$

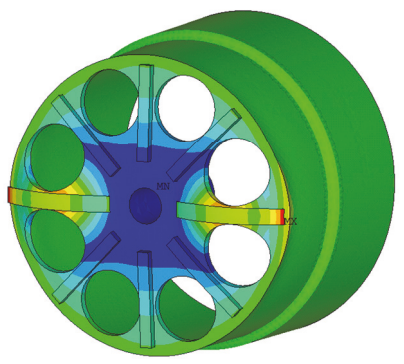

$600 \mu \mathrm{m}$

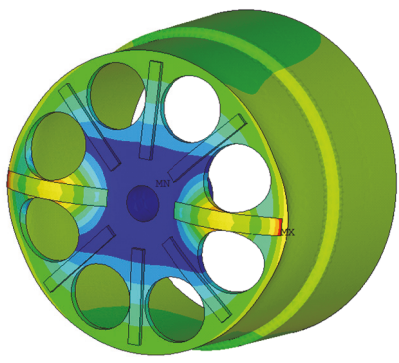

$400 \mu \mathrm{m}$

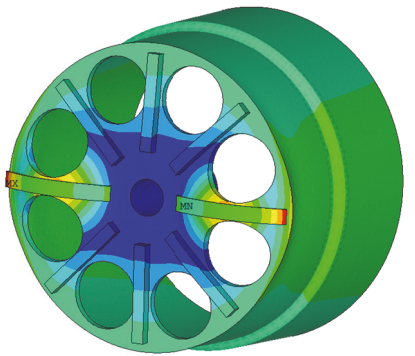

$800 \mu \mathrm{m}$

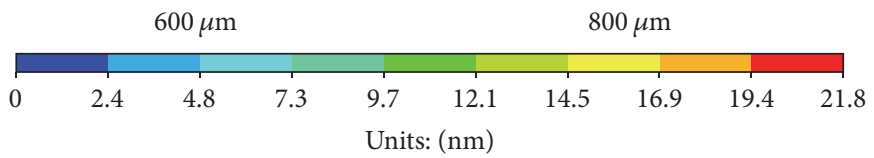

FIgURE 3: The selected finite element simulation results of vibrational amplitudes actuated by piezoelectrodes with different thickness.

of the electrodes. The resonant frequencies were obtained by modal analysis. In order to simplify the simulation process, the deformations were obtained by static analysis. Since the vibration amplitude is proportional to the static deformation at the resonant frequency, this static deformation can be a direct indicator of the vibration amplitude.

Results are shown in Table 3 and Figure 3. And the results in Table 3 are compared with experimental results in Figure 8. The resonant frequency increased slightly with the increase of the electrode thickness, and the change of the resonant frequency is linear, basically. Thicker piezoelectrodes introduced bigger relative changes (defined as $\left(f_{1}-\right.$ $\left.f_{0}\right) / f_{0}$, where $f_{0}$ and $f_{1}$ are the wineglass mode frequency before and after the attachment of piezoelectrodes, resp.).
The vibrational amplitude (evaluated at the bottom edge) was inversely proportional to the electrode thickness, that is, the thinner the piezoelectrodes are, the greater the amplitude is. This is because, under the same driving voltage, the thinner the piezoelectrodes are, the stronger the electric field in the piezoelectrodes is, and the more obvious the inverse piezoelectric effect is, which leads to a greater amplitude. That means the vibrational signal can be easier to detect and achieve a better SNR.

3.2. Experiments. Four brass resonators were fabricated to verify the simulation results. Piezoelectrodes in four different thicknesses were attached on the bottom of the resonators by epoxy resin adhesive, as shown in Figure 4. The thicknesses 
TABLE 2: Dimensions of resonators.

\begin{tabular}{lc}
\hline Devices & Geometric dimension $(\mathrm{mm})$ \\
\hline$b$ & 0.6 \\
$s_{1}$ & 3 \\
$s_{2}$ & 6 \\
$h_{1}$ & 5.5 \\
$r_{1}$ & 0.3 \\
$r_{2}$ & 1 \\
$l_{1}$ & 6 \\
$l_{2}$ & 8 \\
$l_{3}$ & 10 \\
$d$ & 24.4 \\
\hline
\end{tabular}

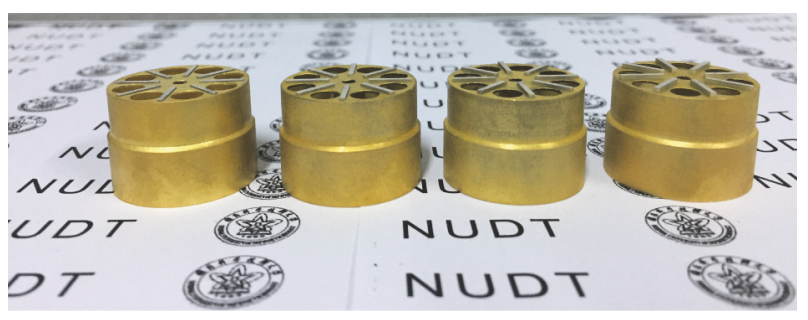

FIGURE 4: Four resonators attached with piezoelectrodes whose thicknesses are $200 \mu \mathrm{m}, 400 \mu \mathrm{m}, 600 \mu \mathrm{m}$, and $800 \mu \mathrm{m}$.

of the piezoelectrodes were $200 \mu \mathrm{m}, 400 \mu \mathrm{m}, 600 \mu \mathrm{m}$, and $800 \mu \mathrm{m}$, respectively.

A laser Doppler vibrometer was used to measure the resonant frequency, amplitude, and $Q$ factor. The resonators were actuated by acoustic sources and by piezoelectric electrodes before and after the attachment of the piezoelectrodes under atmospheric pressure, respectively. The measurement system is shown in Figure 5.

Before the measurement, the actuation direction and the principle axes should be aligned in the same orientation. Then, the vibrational shape is obtained through the scanning of the measurement point defined on the bottom of the resonator, as shown in Figure 6. The antinode of the vibrational shape is selected as the subsequent measurement point. The frequency sweeping data was recorded, and the resonant frequency and amplitude were obtained through fast Fourier transform. The decay time is obtained from the exponential fitting to ring-down data through MATLAB, as shown in Figure 7. The fitting function is as follows:

$$
A(t)=X \cdot \exp \left(-\frac{t}{\tau}\right)
$$

Experimental results are shown in Table 4. And they are compared with simulation results in Figure 8. For electrode thicknesses of $200 \mu \mathrm{m}, 400 \mu \mathrm{m}, 600 \mu \mathrm{m}$, and $800 \mu \mathrm{m}$, the relative increases of the resonant frequencies are $0.14 \%$, $0.24 \%, 0.29 \%$, and $0.36 \%$, respectively, which indicates that thinner piezoelectrodes introduce less change in resonant frequency. Under the same driving voltage $(10 \mathrm{~V})$, the vibrational amplitude is $34.18 \mathrm{~nm}, 22.38 \mathrm{~nm}, 16.47 \mathrm{~nm}$, and $12.08 \mathrm{~nm}$,

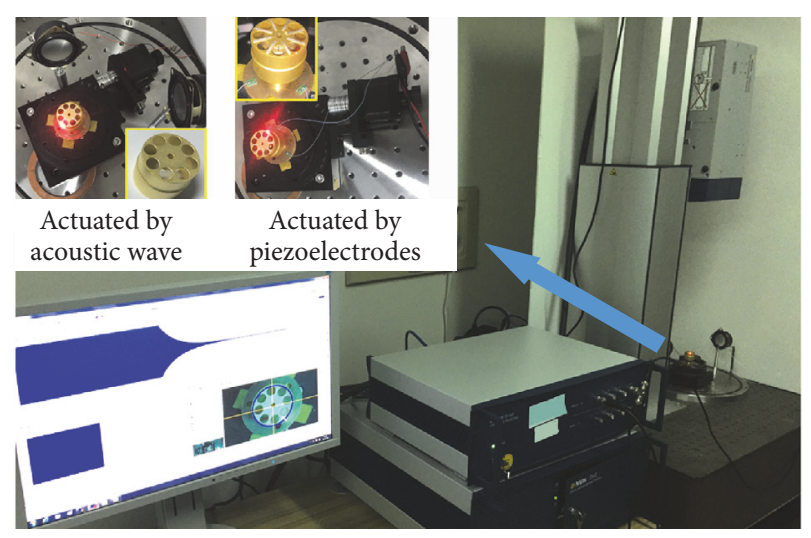

FIGURE 5: The experimental system.

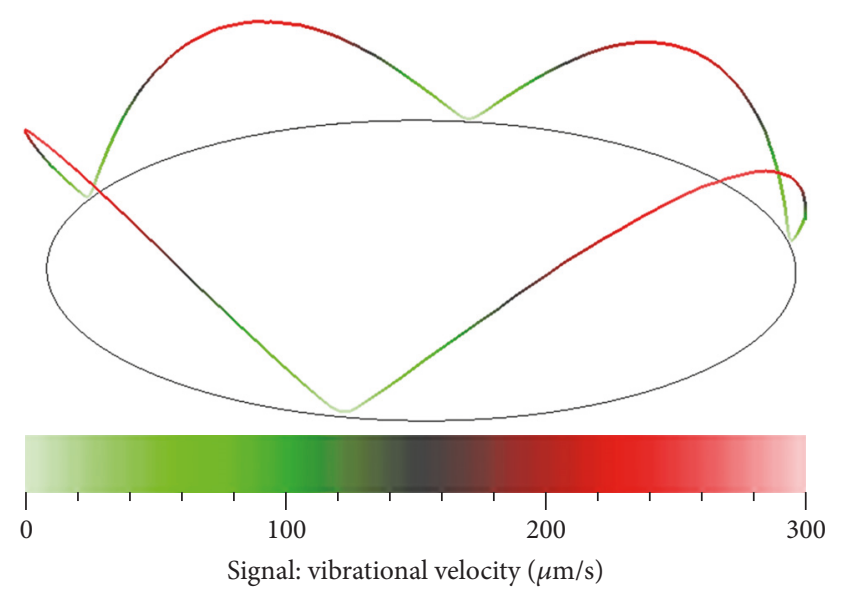

FIGURE 6: The graph of the vibrational shape.

respectively, which shows that thinner piezoelectrodes actuated the resonator to a larger vibrational amplitude. The reduction rate of the $Q$ factor is $9.49 \%, 15.71 \%, 20.65 \%$ and $21.43 \%$, respectively, which indicate that thinner piezoelectrodes are also advantageous in maintaining a higher $Q$ factor.

3.3. Comparison between Simulations and Experiments. The trend of experimental results of the resonant frequency is consistent with the simulation results, as shown in Figure 8. Basically, the change of the resonant frequency is linear. The resonant frequency measured is slightly different compared with the simulation results. This is due to the variation of parameters in practical materials and geometric error in the manufacturing of resonators and electrodes. The trend of experimental results of vibrational amplitude is consistent with the simulation results. It should be pointed out that experimental results of vibrational amplitudes and simulation results of them are not exactly equivalent. The simulation results are calculated under the static condition, whereas experimental results are measured under resonant condition. According to this, the experimental amplitudes are higher than the simulation results. 
TABLE 3: The simulation results of resonant frequencies and vibrational amplitudes under different thicknesses of piezoelectrodes.

\begin{tabular}{lccc}
\hline Thickness $(\mu \mathrm{m})$ & \multicolumn{2}{c}{ Amplitude $(\mathrm{nm})$} \\
0 & 2981.9 & $\begin{array}{c}\text { Resonant frequency }(\mathrm{Hz}) \\
\text { Relative change }\end{array}$ & 19.1 \\
100 & 2984.3 & $0.08 \%$ & 12.4 \\
200 & 2986.2 & $0.14 \%$ & 8.92 \\
300 & 2988.2 & $0.21 \%$ & 8.38 \\
400 & 2990.3 & $0.28 \%$ & 7.93 \\
500 & 2992.3 & $0.35 \%$ & 7.46 \\
600 & 2994.1 & $0.41 \%$ & 6.95 \\
700 & 2995.8 & $0.47 \%$ & 6.70 \\
800 & 2997.3 & $0.52 \%$ & \\
\hline
\end{tabular}

TABLE 4: The comparison of experimental results between before and after the piezoelectrodes were attached.

\begin{tabular}{lccccccc}
\hline \multirow{2}{*}{ Thickness of piezoelectrodes $(\mu \mathrm{m})$} & \multicolumn{3}{c}{ Resonant frequency $(\mathrm{Hz})$} & \multirow{2}{*}{ Vibrational amplitude $(\mathrm{nm})$} & \multicolumn{2}{c}{$Q$ factor } \\
& Before & After & Relative change & & Before & After & Reduction rate \\
\hline 200 & 2720 & 2724 & $0.14 \%$ & 34.18 & 5713 & 5171 & $9.49 \%$ \\
400 & 2816 & 2823 & $0.24 \%$ & 22.38 & 6051 & 5101 & $15.71 \%$ \\
600 & 3038 & 3047 & $0.29 \%$ & 16.47 & 6879 & 5458 & $20.65 \%$ \\
800 & 3026 & 3037 & $0.36 \%$ & 12.08 & 6968 & 5475 & $21.43 \%$ \\
\hline
\end{tabular}

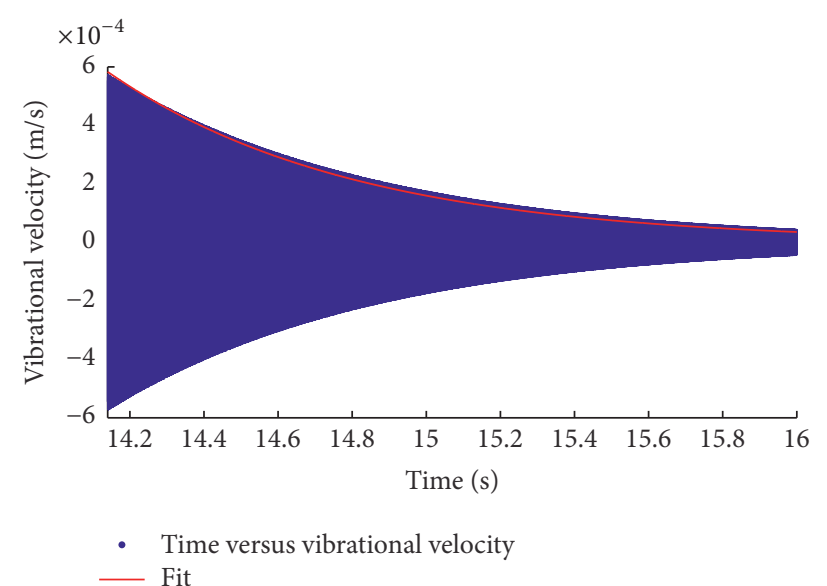

FIgURE 7: The exponential fitting of vibrational amplitude data.

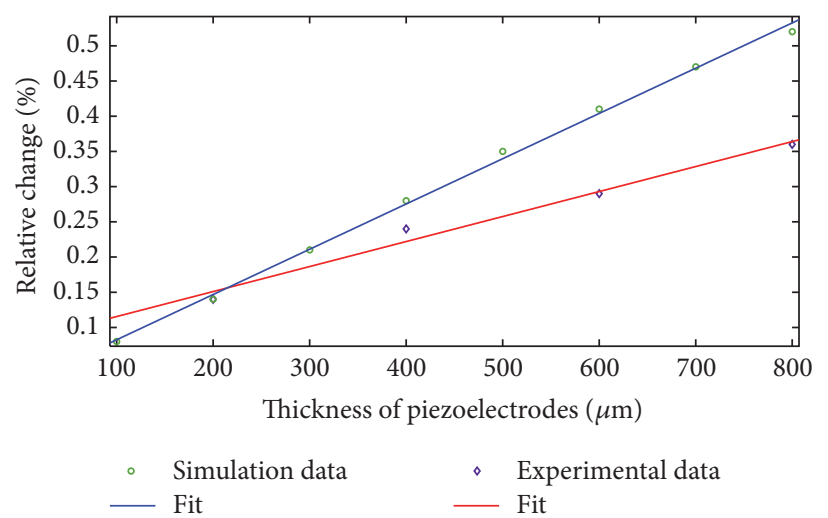

(a) Resonant frequencies

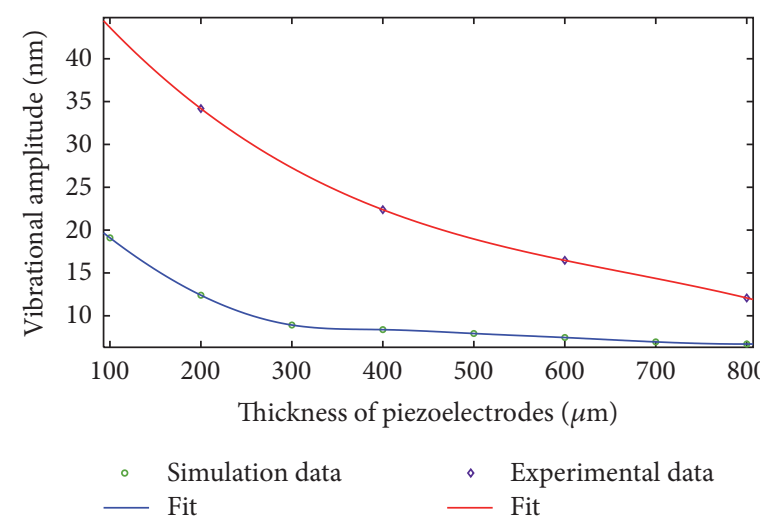

(b) Vibrational amplitudes

FIGURE 8: Comparison of the relative change of resonant frequencies and vibrational amplitudes under different thickness of piezoelectrodes between experiments and simulations. 


\section{Conclusions}

This paper investigated the influence of the electrode thickness on the vibrational properties of a cylindrical shell resonator used in cylindrical resonator gyroscopes. A finite element model was built to simulate the resonator actuated by piezoelectrodes with varied thicknesses under the same driving voltage. The resonant frequencies and vibration amplitudes were calculated. Then experiments were carried out on four resonators with four different electrode thicknesses. Experimental results were consistent with simulations. It was demonstrated that the resonant frequency increases slightly and linearly with the increase of electrode thicknesses. Under the same voltage, thinner electrodes provide larger amplitudes, while introducing less degradation of $Q$ factors. Therefore, it was advantageous to use thinner piezoelectrodes in the actuation of CVG resonators for better noise and drift performance.

\section{Conflicts of Interest}

The authors declare that there are no conflicts of interest regarding the publication of this paper.

\section{Acknowledgments}

This research is supported by the National Science Foundation of China (Grants nos. 11304384 and 61575220). The authors would like to thank the people who helped in mechanical processing.

\section{References}

[1] D. M. Rozelle, "The hemispherical resonator gyro: from wineglass to the planets," Advances in the Astronautical Sciences, vol. 134, pp. 1157-1178, 2009.

[2] V. V. Chikovani, I. M. Okon, A. S. Barabashov, and P. Tewksbury, "A set of high accuracy low cost metallic resonator CVG," in Proceedings of the 2008 IEEE/ION Position, Location and Navigation Symposium (PLANS '08), pp. 238-243, Monterey, CA, USA, May 2008.

[3] V. V. Chikovani, Y. A. Yatsenko, A. S. Barabashov, P. I. Marusyk, E. O. Umakhanov, and V. N. Taturin, "Improved accuracy metallic resonator CVG," IEEE Aerospace and Electronic Systems Magazine, vol. 24, no. 5, pp. 40-43, 2009.

[4] D. Titterton and J. Weston, "Modern inertial navigation technology and its application," Electronics \& Communication Engineering Journal, vol. 12, no. 2, pp. 49-64, 2000.

[5] V. Chikovani and Y. Yatzenko, Coriolis force gyroscope with high sensitivity[P], United States Patent: 7281425B2, 2008.

[6] Y. A. Yatsenko and J. F. Petrenko, "Technological aspects of manufacturing of compound hemispherical resonators for small-sized vibratory gyroscopes," in Proceedings of the Integrated Navigation Systems, RTO SCI International Conference, pp. 71-75, Petersburg, Russia, May 1999.

[7] Waston Industries, 2016, http://watson-gyro.com/product/rategyros/pro-gyro-rate-gyro.

[8] Innalabs, 2016, http://www.innalabs.com/en/products/gyroscopes/.
[9] V. F. Zhuravlev, “Theoretical Foundation of Hemispherical Resonator Gyro (HRG)," Izv. RAN, Mekhanika Tverdogo Tela, vol. 28, no. 3, pp. 3-15, 1993.

[10] Y. K. Zhbanov, "Theoretical aspects of balancing the hemispherical resonator gyro," in Proceedings of the 2nd Saint Petersburg International Conference on Gyroscopic Technology and Navigation, vol. 88, 1995.

[11] F. V. Zhuravlev and D. D. Lynch, "Electric model of a hemispherical resonator gyro," Mechanics of Solids, vol. 30, no. 5, pp. 10-21, 1995.

[12] D. A. Kovriguine, "Geometrical nonlinearity stabilizes a wave solid-state gyro," Archive of Applied Mechanics, vol. 84, no. 2, pp. 159-172, 2014.

[13] E. I. Green, “The story of Q, American Scientist, pp. 584-594, 1955.

[14] Y. Pan, D. Wang, Y. Wang et al., "Monolithic cylindrical fused silica resonators with high Q factors," Sensors, vol. 16, no. 8, article 1185, 2016.

[15] F. Goldschmidtboeing and P. Woias, "Characterization of different beam shapes for piezoelectric energy harvesting," Journal of Micromechanics and Microengineering, vol. 18, no. 10, Article ID 104013, 2008.

[16] J. E. Loper and D. D. Lynch, "Sonic vibrating bell gyro," Sonic vibrating bell gyro, U.S. Patent 4,157,041[P], 1979-6-5.

[17] D. Lynch and A. Matthews, "Dual-mode hemispherical resonator gyro operating characteristics," in Proceedings of the 3rd Saint Petersburg International Conference on Integrated Navigation Systems, pp. 37-44, 1996.

[18] E. J. Loper and D. D. Lynch, "Projected system performance based on recent HRG test results (low noise inertial rotation sensor)," in Proceedings of the IEEE/AIAA 5th Digital Avionics Systems Conference, p. 18, 1983.

[19] D. M. Rozelle, "The hemispherical resonator gyro: from wineglass to the planets," in Proceedings of the 19th AAS/AIAA Space Flight Mechanics Meeting, pp. 1157-1178, 2009.

[20] Y. Tao, Study on key technologies of cupped wave [pdf thesis], National University of Defense Technology, Changsha, China, 2011.

[21] J. Soderkvist, "Piezoelectric beams and vibrating angular rate sensors," IEEE Transactions on Ultrasonics, Ferroelectrics, and Frequency Control, vol. 38, no. 3, pp. 271-280, 1991.

[22] H. Uehara, T. Ohtsuka, T. Inoue, M. Yoshimatu, H. Matudo, and M. Okazaki, "Miniaturized angular rate sensor with laminated quartz tuning fork," in Proceedings of the 2005 Joint IEEE International Frequency Control Symposium (FCS) and Precise Time and Time Interval (PTTI) Systems and Applications Meeting, pp. 886-891, August 2005.

[23] P. W. Loveday, "Analysis of piezoelectric ulstraonic transducers attached to waveguides using waveguide finite elements," IEEE Transactions on Ultrasonics, Ferroelectrics, and Frequency Control, vol. 54, no. 10, pp. 2045-2051, 2004.

[24] T. L. Floyd, Electric Circuits Fundamentals, Prentice hall, New Jersey, NJ, USA, 2009.

[25] B. Zhu, Y. Tao, Y. Wu, and Z. Chen, "Research on adhensive layer and its effects on vibtation characteristics of resonator and PZT of cupped gyro," Chinese Journal of Sensors and Actuators, vol. 24, no. 9, pp. 1248-1252, 2011. 


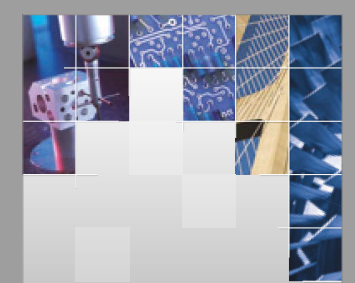

\section{Enfincering}
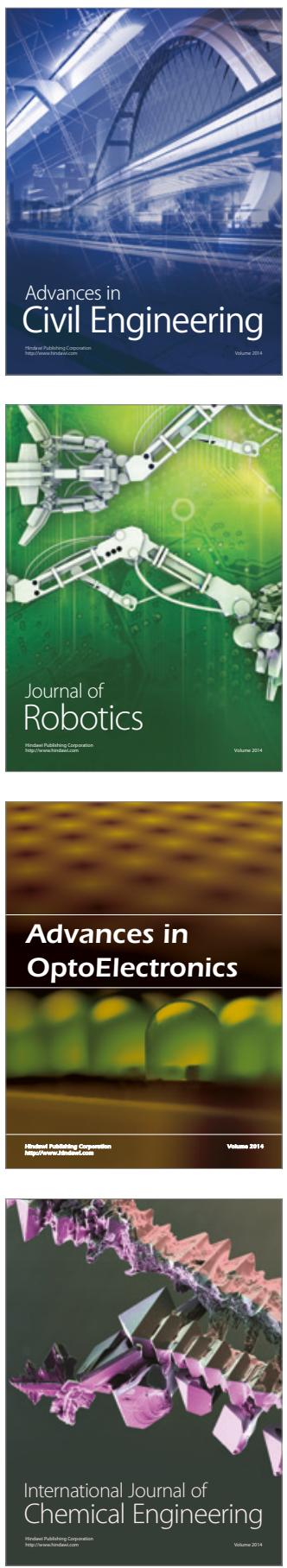

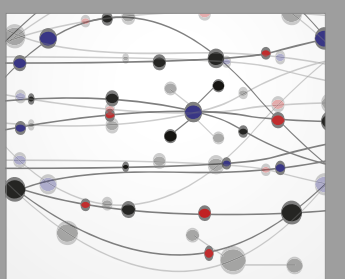

The Scientific World Journal

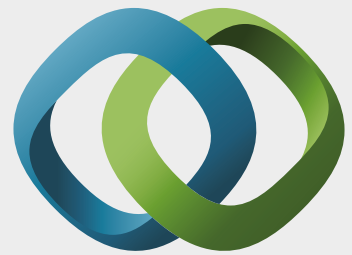

\section{Hindawi}

Submit your manuscripts at

https://www.hindawi.com
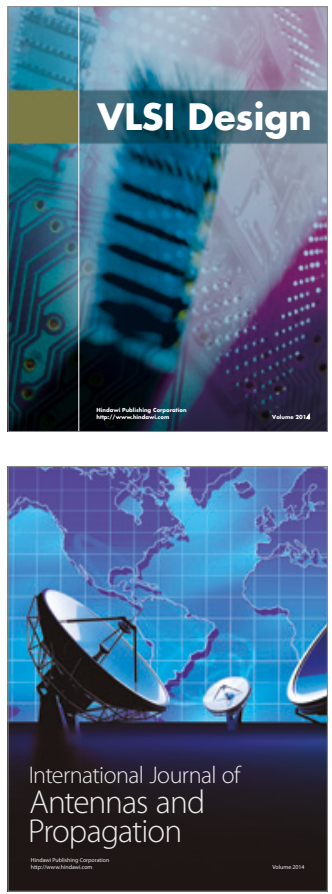

\section{Rotating}

Machinery
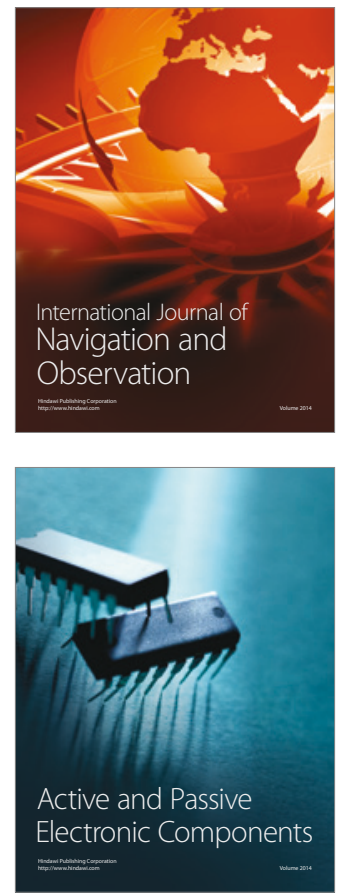
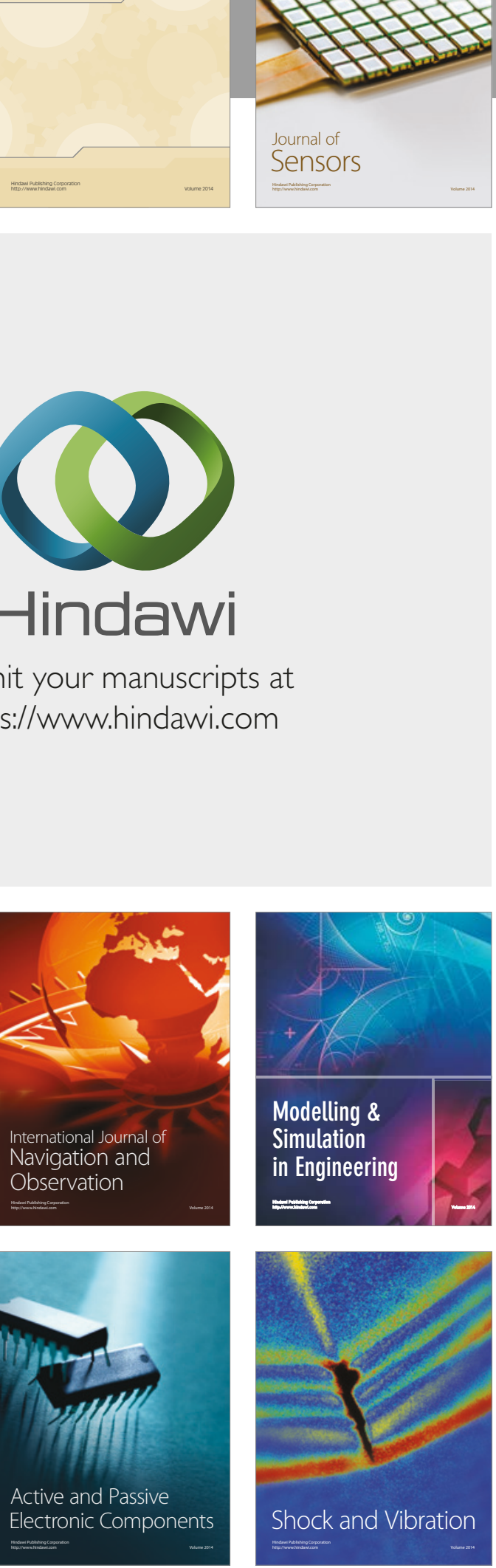
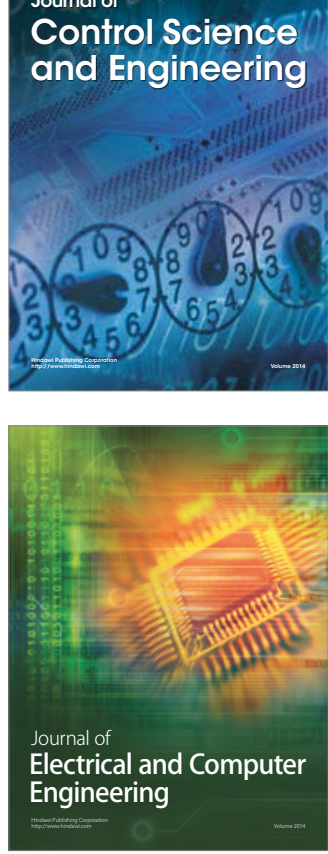

Distributed

Journal of

Control Science

and Engineering
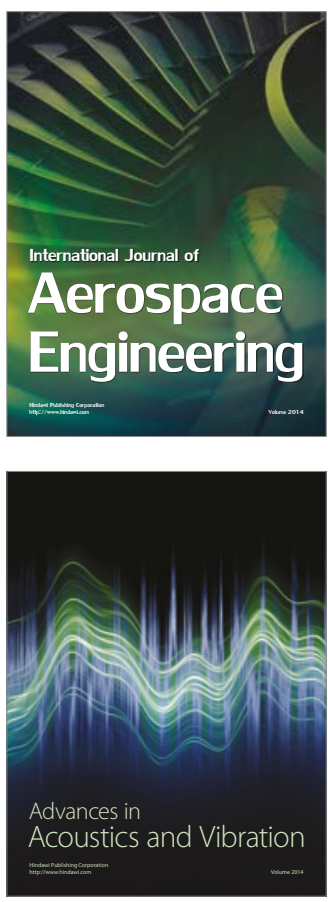

Sensor Networks 